Toll-like receptor 1 (TLR1) recognises lipopeptides with TLR2, and affects immune response to Mycobacterium tuberculosis infection. Here, we report results of the first case-control paediatric study of the TLR1 single-nucleotide polymorphisms and susceptibility to tuberculosis (TB). A paediatric case-control study enrolled $340 \mathrm{~TB}$ patients and 366 healthy controls, all Han Chinese from North China. Significant differences of the allelic and genotypic distributions of rs5743618 in TLR1 gene were observed between TB group and control group and, G allele of rs5743618 was associated with increased risk for TB (OR: 2.40, 95\% CI: $1.41-4.07, \mathrm{p}=0.0009)$. In addition, after stimulated with inactivated lysate of Mycobacterium tuberculosis strain H37Rv, whole blood samples from children with the rs5743618 GT genotypes showed a decreased level of Interleukin-12p40 (IL-12p40), Tumour Necrosis Factor-a (TNF-a) and CXC chemokine ligand 10 (CXCL10) production. To conclude, TLR1 rs5743618 G allele was found associated to susceptibility to TB in Han Chinese paediatric population. TLR1 rs5743618-GT genotype carriers may have reduced immune response to MTB infection although further study is warranted to test this conclusion.

\section{PS-327 PARENTS: THE BEST PARTNER IN PAIN MANAGEMENT}

MGE Jansen, CJ van der Perk, JM Maaskant, L van der Maat, B Brouwer. Women's and Children's Clinic, Academic Medical Center, Amsterdam, Netherlands

\subsection{6/archdischild-2014-307384.626}

Introduction Infants in hospital experience pain regularly. It is an important nursing task to observe parameters of pain, because an infant is not able to express itself verbally. The question arises in which way and to what extent parents can play a role in recognising and diminishing pain experienced by their child.

Method Involvement of parents in pain management has been investigated by means of a literature search and a survey among nurses. We also explored how parents' involvement could be increased in an effective way.

Results Research shows that parents' ínvolvement in pain management is effective. When parents were given an active role, there was a quicker response to pain signals and the child experienced less pain.

Parents' involvement is also feasible. A structured method of providing information increased knowledge of parents. In addition to oral information, demonstrations and videos were of extra value. However, in daily practice parents' involvement in pain management is limited. Parents did not always receive oral $(55 \%)$ or written $(4 \%)$ information about pain management.

Nurses indicated more parents' involvement than is experienced by parents themselves.

The survey showed that $96 \%$ of nurses believe that parents are actively involved in pain recognition and $85 \%$ thinks that parents play an active role in pain reduction. However, only
Abstract PS-328 Table $1{ }^{+}$T cell epitopes from PPE proteins of M.tuberculosis predicted by using Net.4 Server

\begin{tabular}{lllllll}
\hline & PPE8 & PPE12 & PPE21 & PPE39 & PPE62 & \\
Alleles of & (Rv0355c) & (Rv0755c) & (Rv1548c) & (Rv2353c) & (Rv3533c) & SB/WB \\
Class I HLA & $3292^{1}$ & 637 & 670 & 346 & 574 & (Total) \\
HLA-A*1101 & $4 / 9^{2}$ & $1 / 1$ & $0 / 1$ & $0 / 2$ & $0 / 1$ & $5 / 14(19)$ \\
HLA-A*2402 & $1 / 7$ & $0 / 1$ & $0 / 3$ & $1 / 0$ & $0 / 1$ & $2 / 12(14)$ \\
HLA-A*0201 & $20 / 43$ & $8 / 4$ & $3 / 9$ & $2 / 1$ & $1 / 8$ & $34 / 65(99)$ \\
HLA-B*4601 & $0 / 2$ & $0 / 0$ & $0 / 1$ & $0 / 0$ & $0 / 1$ & $0 / 4(4)$ \\
HLA-B*4001 & $5 / 3$ & $1 / 1$ & $1 / 2$ & $0 / 0$ & $0 / 2$ & $7 / 8(15)$ \\
HLA-B*5101 & $0 / 6$ & $0 / 0$ & $0 / 1$ & $0 / 0$ & $0 / 1$ & $0 / 8(8)$ \\
SB/WB (Total) & $30 / 70(100)$ & $10 / 7(17)$ & $4 / 17(21)$ & $3 / 3(6)$ & $1 / 14(15)$ & $48 / 111(159)$ \\
\hline
\end{tabular}

$53 \%$ of the nurses think that parents are actually able to decrease pain.

Conclusion Parents' participation in pain recognition and pain reduction is effective. Parents can be trained by means of structured methods of information, demonstrations and videos.

\section{PS-328 PREDICTION OF HUMAN PROMISCUOUS MHC CLASS I RESTRICTED CD8 ${ }^{+}$T-CELL EPITOPES IN THE PPE PROTEIN FAMILY OF MYCOBACTERIUM TUBERCULOSIS: A COMPUTATIONAL APPROACH}

J Xiao, A Shen, L Sun, W Jiao, C Shen. Tuberculosis Research Laboratory, Bei Jing Children's Hopspital, Beijing, China

\subsection{6/archdischild-2014-307384.627}

Background and aims Tuberculosis (TB), caused by infection with Mycobacterium tuberculosis, is a major cause of morbidity and mortality worldwide. Although the wide use of Mycobacterium bovis bacille Calmette-Guérin (BCG), the true effectiveness of BCG vaccine has been debated for decades. Others, the sensitivity of both tuberculin skin test and IFN- $\gamma$-release assays is suboptimal, and none of these tests distinguish between latent infection and active disease. So, there is a pressing need to detect new TB antigens to develop effective vaccines capable of activating the immune responses relevant for protection and to set up sensitive immunological tests that may improve the identification of latent TB.

Methods In this study, through database access to the DNA and protein sequences of PPE proteins and the use of bioinformatics programs, including SignalP4.1 server, SecretomeP 2.0 server, DAS server and NetMHC3.4 server, promiscuous epitope peptides were identified.

Results We identified four promiscuous epitope peptides. Those four peptides can bind to more than two HLA molecules. Three peptides were obtained from PPE8 protein and one from PPE12 protein. PPE8 might turn out as a useful reagent for TB subunit vaccines and diagnostic antigens.
Abstract PS-328 Table 2 Distribution of promiscuous ${ }^{+} \mathrm{T}$ cell epitopes

\begin{tabular}{llll}
\hline Peptide & $\begin{array}{l}\text { Proteins on which the epitope } \\
\text { polypeptide located }\end{array}$ & $\begin{array}{l}\text { Aminoacid sequences of } \\
\text { epitope polypeptide }\end{array}$ & $\begin{array}{l}\text { Sites of epitope polypeptide } \\
\text { (Length) }\end{array}$ \\
p1 & PPE8 (Rv0355c) & RLAAAAFEALAATVHPA & P90-107 (18aa) \\
p2 & PPE8 (Rv0355c) & GTFTVHGFRFPEITGDIFLIGIPFNA & P1760-1785 (26aa) \\
p3 & PPE8 (Rv0355c) & ALGVTHFSVGPIIVPR & P2261-2276 (16aa) \\
p4 & PPE12 (Rv0755c) & FEEALAGVVHPA & P97-108 (12aa) \\
\hline
\end{tabular}


A SignalP-4.1 prediction (gram+ networks): Sequence

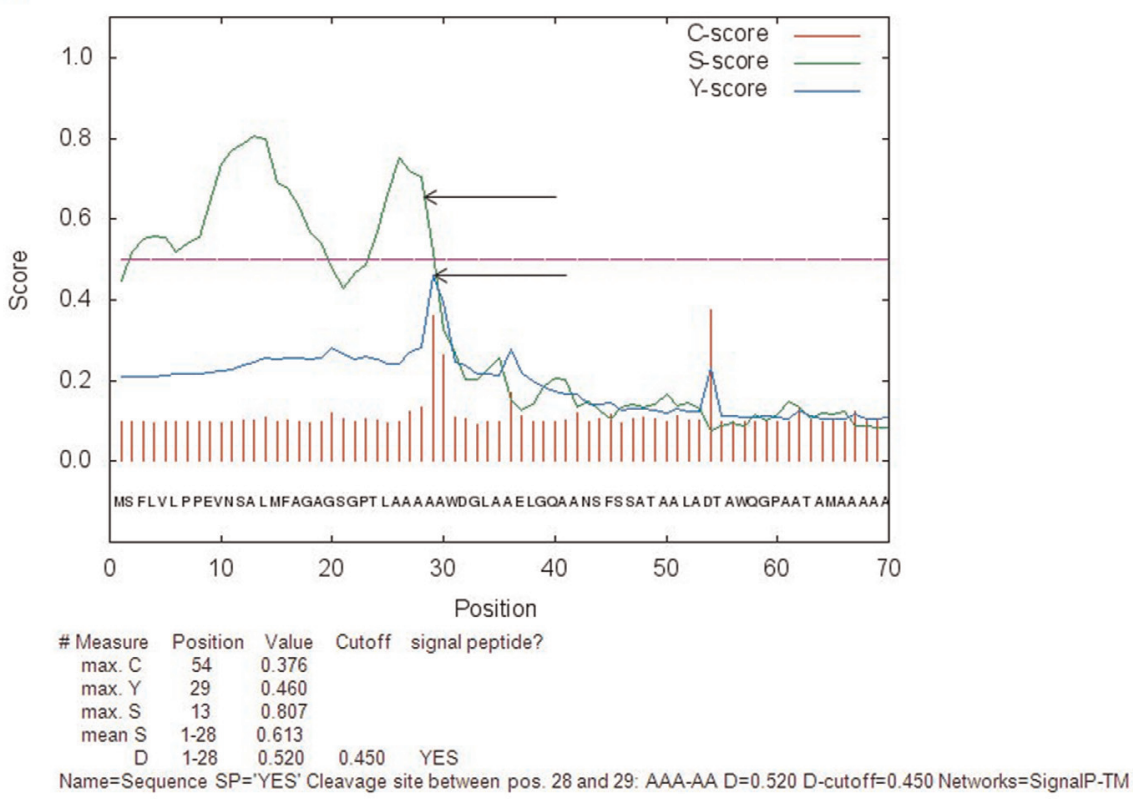

B SignalP-4.1 prediction (gram+ networks): Sequence

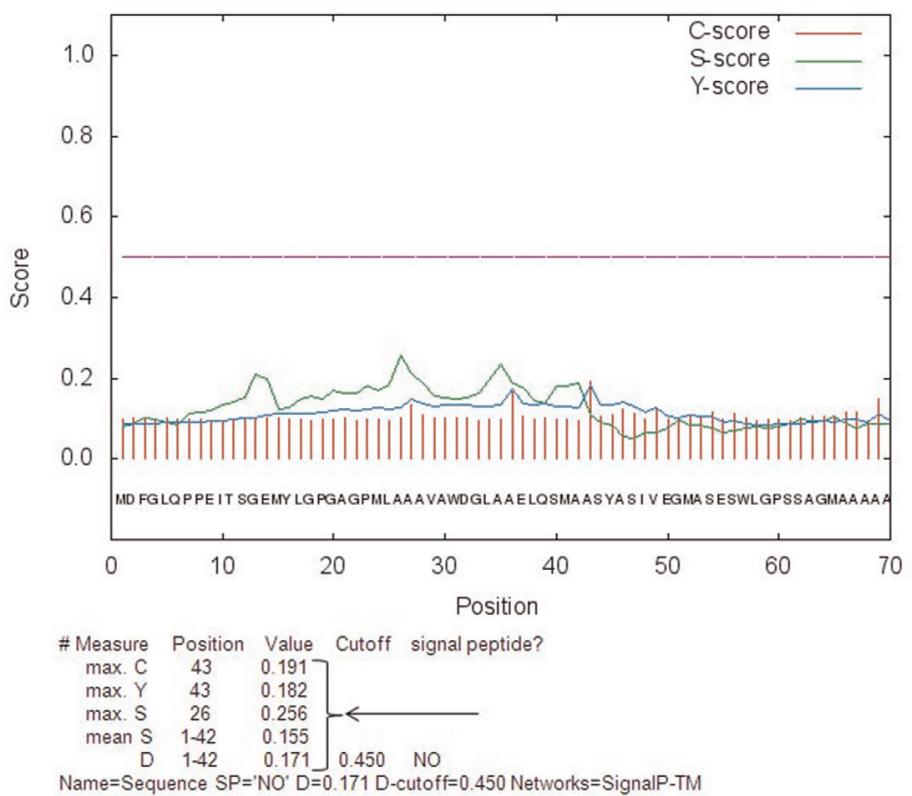

Abstract PS-328 Figure 1 Signal peptide prediction. (A)The presence of a signal peptide in protein PPE16, where S-mean indicates the amino acids that are most likely to compose the signal peptide, and Y-max indicates the cleavage site. (B) No signal peptide was found in protein PPE33, as indicated by arrow.

Conclusions Prediction analysis showed that these promiscuous epitope peptides presented here may be important targets in the search for subunit vaccines or diagnostic antigens against Mycobacterium tuberculosis. Additionally, we suggest that these peptides may be used in immunological assays to evaluate the level of protection, the effect on pathology reduction and the profile of cytokines and antibodies induced by them.

Note: 1 represents the total number of the epitopes of each protein; 2 the left of the solidus represents the number of strong-bind epitopes; the right represents the number of weakbind epitopes.

\section{PS-329 TLR3 MEDIATED EXPRESSIONS OF CYTOKINES AND SOCS1 IN RESPIRATORY SYNCYTIAL VIRUS PERSISTENT CELLS}

J Zheng, PU Yang, D Zhao. Pediatrics, Zhongnan Hospital of Wuhan University, Wuhan, China

\subsection{6/archdischild-2014-307384.628}

Background Respiratory syncytial virus (RSV) persistent HEp-2 cells are a heterogeneous mixture of viral antigen-positive and negative variants, and the mechanism through which viral 